\title{
Ethnicity may be linked to thin body preoccupation and social pressure in the development of eating disorders
}

The McKnight Investigators. Risk factors for the onset of eating disorders in adolescent girls: results of the McKnight

Longitudinal Risk Factor Study. Am J Psychiatry 2003 Feb;160:248-54.

\section{QUESTION: What are the risk factors for eating disorders in adolescent girls?}

\section{Design \\ Cohort study.}

\section{Setting}

Two schools in Arizona and California, USA; timeframe not specified.

\section{Participants}

1,103 girls initially assessed in grades $6-9.39 \%$ were white, $13 \%$ black and $34 \%$ Hispanic. There was a significant difference between the proportion of black and white students enrolled at the two different schools.

\section{Intervention}

Students completed the McKnight Risk Factor Survey, had their height and weight measured, and participated in a structured clinical interview annually for 4 years.

\section{Main outcome measures}

Eating disorders and associated risk factors, measured by clinical interview and the McKnight Risk Factor Survey, a self-report questionnaire developed for adolescents. The instrument, with data on norms, psychometrics, and test-retest reliabilities, is available at http://bml.stanford.edu.

\section{Main results}

During the follow up period, $2.9 \%$ of the participants (32 girls) developed partial or full syndrome eating disorder. Factors associated with eating disorders were Hispanic ethnicity, thin body preoccupation, self reported dieting, social pressure, and an increase in negative life events.

\section{Conclusions}

A preoccupation with having a thin body and social pressure are risk factors for developing eating disorders in adolescent girls. Some Hispanic groups are particularly at risk of eating disorders. The authors suggest that efforts to reduce peer, cultural, and other sources of thin body preoccupation may be important for reducing eating disorders.

\section{COMMENTARY}

Several features make this an outstanding investigation into the risk factors for eating pathology. The authors used a prospective design with a long follow up period, structured diagnostic interviews for case ascertainment, a large ethnically diverse sample, powerful analytic techniques, and reported effect sizes.

There are some caveats, however. (1) The authors used a new measure to assess putative risk factors, rather than established scales with greater evidence of reliability and construct validity; (2) The atheoretical data reduction approach did not account for the possibility that some risk factors may mediate the effects of others. The scales resulting from data reduction were amalgams of conceptually distinct constructs (for instance, body dissatisfaction and depressed mood). This makes it difficult to interpret results; (3) The overall attrition rate was high, which also limits generalisability.

Taken in the context of the larger evidence-base, the findings have several theoretical and clinical implications. Findings about the predictive status of internalising the thin ideal and perceived pressure to be thin are similar to past studies. This suggests that interventions that make girls more resilient to these pressures may help prevent eating disorders. Similarly, the predictive status of psychopathology and body dissatisfaction reinforce past findings. This implies that interventions that reduce mood disturbances and body image concerns may reduce the risk for eating pathology.

Although the predictive finding for self reported dieting has been reported previously, observational data from four studies suggest that such measures do not reflect true dietary restriction. Experiments have found that placing individuals on low calorie diets actually reduces eating pathology. These results imply that dietary restriction is not a causal risk factor for eating pathology, as is widely assumed. The McKnight study found that girls who alter their eating behaviours for impression management purposes are at greater risk for eating pathology. This might suggest that a general propensity towards overeating is the true risk factor, which leads girls to diet and alter their public eating behaviour in an effort to curb this tendency.

These conflicting findings illustrate the importance of collecting data on the validity of self report measures. They also serve to remind researchers and clinicians that third variable confounds in longitudinal studies may explain prospective effects. This underscores the importance of confirming prospective findings with randomised trials that manipulate ostensive risk factors.

Eric Stice, $\mathrm{PhD}$ University of Texas Austin, Texas, USA urce of funding: McKnight Foundation $\mathrm{NIMH}$

For correspondence: CB Taylor, Department of Psychiatry, Stanford University School of Medicine, California, USA.

btaylor@stanford.edu 